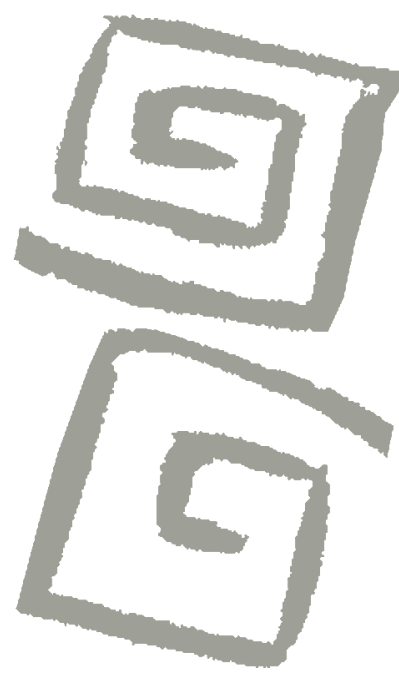

\title{
Análisis cualitativo del deterioro de la calidad del agua y la infección por Helicobacter pylori en una comunidad de alto riesgo de cáncer de estómago (Cauca, Colombia)
}

\author{
Qualitative analysis of water quality deterioration and \\ infection by Helicobacter pylori in a community with \\ high risk of stomach cancer (Cauca, Colombia)
}

Acosta, Claudia Patricia'; Benavides, John Alexander²; Sierra, Carlos Hernán ${ }^{3}$

${ }^{1}$ Doctora en Ciencias Ambientales. Profesora, investigadora, Laboratorio de Genética Humana, Departamento de Ciencias Fisiológicas, Facultad de Ciencias de la Salud Universidad del Cauca, Popayán, Colombia. cpacosta@unicauca.edu.co

${ }^{2}$ Magíster en Salud y Seguridad en el Trabajo. Docente Investigador, Universidad EAN, Bogotá, Colombia. johnbena1@gmail.com

${ }^{3}$ Doctor en Epidemiología Molecular. Profesor,

Laboratorio de Genética Humana, Departamento de Ciencias Fisiológicas, Facultad de Ciencias de la Salud, Universidad del Cauca,

Popayán, Colombia.

hsierraphd@gmail.com
RESUMEN Este estudio aborda aspectos de la salud ambiental de la población rural de Timbío (Cauca, Colombia) con relación al deterioro de la calidad del agua. La información se obtuvo mediante métodos de investigación participativa, relacionadas al manejo y uso del agua, fuentes de contaminación y percepción de la calidad del agua y su relación con la infección por Helicobacter pylori. Los resultados forman parte del análisis cualitativo de una primera fase de caracterización de la problemática hídrica y sanitaria y su relación con enfermedades infecciosas emergentes y soluciones comunes, llevada a cabo entre noviembre de 2013 y agosto de 2014. Los resultados de esta investigación se discuten desde un enfoque ecosistémico de la salud humana, que reconoce la complejidad de los conflictos ambientales relacionados con el recurso hídrico y su impacto en la salud de las poblaciones. A través de la metodología empleada, este estudio permitió conocer y visualizar los problemas prioritarios y las causas frecuentes de la contaminación del recurso hídrico, para postular algunas soluciones a través de una agenda compartida con los actores sociales.

PALABRAS CLAVES Helicobacter pylori; Percepción; Abastecimiento de Agua; Colombia.

\begin{abstract}
This study looks at aspects of the environmental health of the rural population in Timbío (Cauca, Columbia) in relation to the deterioration of water quality. The information was obtained through participatory research methods exploring the management and use of water, the sources of pollution and the perception of water quality and its relation to Helicobacter pylori infection. The results are part of the qualitative analysis of a first research phase characterizing water and sanitation problems and their relation to emerging infectious diseases as well as possible solutions, which was carried out between November 2013 and August 2014. The results of this research are discussed from an ecosystemic approach to human health, recognizing the complexity of environmental conflicts related to water resources and their impacts on the health of populations. Through the methodology used, it is possible to detect and visualize the most urgent problems as well as frequent causes of contamination of water resources so as to propose solutions within a joint agenda of multiple social actors.
\end{abstract}

KEY WORDS Helicobacter pylori; Perception; Water Supply; Colombia. 


\section{INTRODUCCIÓN}

El hombre, como parte del ecosistema, ha mantenido fuertes relaciones dinámicas con el ambiente (1). Estas relaciones han posibilitado el establecimiento de las comunidades, sus comportamientos sociales, culturales y el desarrollo económico. Sin embargo, existe una creciente crisis del medio ambiente como consecuencia del modelo de producción y su influencia negativa en la salud de los ecosistemas y de los seres humanos.

El proceso de salud-enfermedad está determinado por interacciones con el medio ambiente, en el marco de estructuras sociales, culturales, económicas y políticas que se organizan en un territorio específico (2). En ese contexto, el modelo de desarrollo y sus consecuencias sobre el medio ambiente se expresan también como impactos en la salud humana. Según la Organización Mundial de la Salud (OMS) la carga global de mortalidad atribuible a las condiciones del ambiente es del $23 \%$ y en los países en desarrollo asciende al $25 \%$ (3). El 3,1\% (1,7 millones) de las muertes a nivel mundial son atribuidas a condiciones de saneamiento ambiental, agua e higiene (4).

En Colombia, se estima que la carga de enfermedad atribuible a las condiciones ambientales es del $17 \%$, con 46.000 muertes al año, lo cual se debe en parte a la inequidad en el acceso a servicios públicos, la falta de ingresos y el crecimiento acelerado del sector industrial, factores que incrementan los niveles de vulnerabilidad y exposición de la población, cuyo impacto negativo genera significativos niveles de carga de enfermedad (5).

Los factores ambientales que más contribuyen a provocar muertes prematuras en Colombia son la contaminación del aire y las condiciones del agua, saneamiento e higiene. A pesar de que Colombia es el sexto país con mayor oferta hídrica en el mundo, el Ministerio de Medio Ambiente calcula que la mitad de los recursos hídricos tienen problemas de calidad. Se estima que la industria, el sector agropecuario y las aguas domésticas generan 9.000 toneladas de materia orgánica contaminante de los acuíferos.

La baja calidad del agua para consumo humano y la gestión inadecuada de las sustancias químicas se convierten en los problemas de más alto costo para la economía colombiana (6): cerca del $70 \%(0,77 \%$ del PIB $)$ es atribuible a los impactos en la salud (mortalidad y morbilidad) y el $30 \%$ restante es atribuible a costos de prevención (consumo de agua embotellada, filtrado y hervido doméstico del agua para su potabilización) (5).

A nivel regional, el departamento de Cauca es uno de los de mayor riqueza hídrica en Colombia, donde las prácticas en el uso y manejo del agua se relacionan con la identidad cultural de las diversas étnias que habitan la región. Sin embargo, de acuerdo con el censo 2005 del Departamento Administrativo Nacional de Estadística (DANE), el $46,4 \%$ de los hogares presenta necesidades básicas insatisfechas. El servicio de alcantarillado no llega ni siquiera a la mitad de los hogares en el área rural y la cobertura del servicio de acueducto en las cabeceras municipales es apenas del $66 \%$. Algunas cabeceras municipales presentan problemas tanto en la continuidad del servicio como en la calidad del agua suministrada y tienen condiciones de saneamiento insuficientes. Este es el caso del municipio de Timbío, donde las enfermedades gastrointestinales, la diarrea, la gastritis, entre otras, son las primeras causas de consulta médica (7). Además, es uno de los municipios con alta frecuencia de infección por patógenos como Helicobacter pylori $(85 \%)$, que se asocia al desarrollo de cáncer gástrico, primera causa de muerte por cáncer en el departamento del Cauca (8). Por lo tanto, podemos decir que las problemáticas hídricas constituyen una expresión manifiesta del estado de la salud ambiental de una región.

Este tipo de problemáticas que vinculan profundamente el medio ambiente, la salud y el trabajo han sido abordadas por investigadores desde enfoques ecosistémicos de la salud (ecosalud) (9-12). Este enfoque ofrece herramientas teórico-conceptuales y metodológicas que involucran acciones de transformación de las condiciones determinantes del deterioro ambiental o de la salud junto con los colectivos involucrados $(9,13)$, por lo que uno de los pilares fundamentales es la participación social (12). Este fenómeno, concebido como una herramienta de transformación social, busca que las acciones de los colectivos contribuyan al mejoramiento de las condiciones que deterioran la salud y el medio ambiente, mediante la inclusión de actores sociales, investigadores y funcionarios públicos en el análisis de los problemas que involucran, además, aspectos políticos y sociales $(12,14)$. 
Por otro lado, para Lebel (12), la participación en el enfoque ecosistémico se establece a través de una aproximación metodológica relacionada con los procesos de investigación como herramienta de transformación social. Bajo este contexto, la participación se expresa como un elemento que forma parte de la identificación y planteamiento del problema, así como también de la evaluación y acción en el marco de la investigación. Esto facilita el planteamiento de trabajos participativos a partir de la interacción creativa entre los diversos actores, favoreciendo el diálogo entre los sistemas de conocimiento que entran en juego durante un proceso de intervención e investigación en ecosalud.

La participación social se convierte, además, en un espacio en el que se privilegian las transformaciones de aspectos políticos, culturales y sociales, con el fin general de la democratización de la sociedad. Este paradigma, construido bajo la investigación-acción participativa (IAP), busca la construcción de conocimiento que les permita a las colectividades comprender su compleja realidad a fin de poder transformarla (15). Desde esta perspectiva, se identifican tres grupos de participantes: los especialistas, académicos y científicos; los actores afectados o interesados; y, finalmente, quienes tienen el poder de decisión en diferentes niveles (formales e informales) (16). Para cada grupo de involucrados, pueden identificarse las relaciones sociales y políticas que se establecen a partir de metodologías participativas y colaborativas, teniendo en cuenta su interés y poder con relación al tema (17); incluso, es posible definir los cambios deseados (alcances), una estrategia para conseguirlos y un método de seguimiento que permita comprobar el progreso alcanzado.

Con este estudio, se pretende promover cambios a partir de evidencias construidas participativamente, entendiendo que la cooperación de los individuos conecta la investigación con la acción, lo cual convierte la participación en un principio central.

Teniendo en cuenta el fracaso de las estrategias basadas en el modelo biomédico para transformar las condiciones determinantes del proceso salud-enfermedad en los colectivos, surge la necesidad de generar acciones conjuntas entre investigadores, actores políticos y organizaciones populares, que pongan en evidencia la situación de las condiciones ambientales, de saneamiento y las percepciones de calidad del agua con el fin de generar acciones sostenibles, Ilevadas a cabo por las comunidades, para la trasformación de la realidad que configuran los procesos salud-enfermedad y su relación con los ecosistemas en el territorio.

Por estas razones, en el marco del proyecto “Uso y manejo del agua y su relación con enfermedades infecciosas emergentes en ocho municipios del Cauca", se realizó una primera fase de investigación cualitativa con el fin de problematizar, desde la articulación de los saberes populares y científicos, las significaciones de las comunidades usuarias de los acueductos, respecto a: fuentes de contaminación y condiciones de saneamiento asociadas al agua, percepción de la calidad del agua, y la relación entre salud, medio ambiente y Estado. Adicionalmente, se buscó generar una agenda política de transformación que permita la garantía integral de los derechos al medio ambiente sano y a la salud en los colectivos.

\section{METODOLOGÍA}

En este estudio se utilizó la metodología de investigación-acción participativa con el objetivo de conocer la situación existente en el área de estudio con relación al deterioro del recurso hídrico y los impactos en la salud en los colectivos, y establecer acciones para transformarla $(5,18,19)$. Se conformó un equipo interdisciplinario al que se incorporaron once profesionales, miembros líderes de la comunidad y de instituciones a nivel local.

El estudio se realizó con las comunidades abastecidas por siete acueductos rurales del municipio de Timbío, que de forma histórica y solidaria han construido y sostenido estos sistemas de abastecimiento (Cuadro 1).

Para lograr una construcción de conocimiento que le permitiera a las colectividades comprender la complejidad de la relación entre salud-trabajo y medio ambiente, y generar acciones de transformación, se diseñaron tres etapas. En la primera, denominada "convocatoria", se llevó a cabo una estrategia de acercamiento comunitario e institucional concertada bajo el tema central "El agua y la salud", en la que se vincularon actores institucionales, organizaciones sociales, populares 
Cuadro 1. Acueductos participantes del municipio de Timbío, Departamento de Cauca, Colombia, entre noviembre de 2013 y agosto de 2014

\begin{tabular}{lrr}
\hline Acueducto & $\begin{array}{c}\text { Familias } \\
\text { abastecidas }\end{array}$ & Usuarios \\
\hline El Saladito & 1.300 & 6.500 \\
Las Cruces & 800 & 4.000 \\
Aires del Campo & 350 & 1.600 \\
Las Yescas & 180 & 900 \\
Brisas del Paramillo & 70 & 350 \\
El Placer & 30 & 120 \\
Distrito de Riego & 1.500 & 307 \\
\hline
\end{tabular}

Fuente: Elaboración propia.

y lideres comunitarios. Esta estrategia buscó la participación activa desde los diferentes ámbitos, con el fin de generar aproximaciones de cada uno de los grupos sociales capaces de expresar las disputas basadas en principios económicos, de desarrollo, de poder y la reivindicación para mejorar las condiciones de vida y del ambiente. Además, se sumaron actores sociales claves, relacionados con la gestión y administración del medio ambiente, el recurso hídrico y la salud, líderes comunitarios y la comunidad en general.

En una segunda etapa, denominada "problematización para la transformación", se llevaron a cabo dos talleres por cada zona abastecida por los acueductos, con el fin de comprender la lógica interna de los colectivos. Para el primer taller, el grupo de investigación diseñó una guía de preguntas (Cuadro 2) que buscaba problematizar tres tópicos fundamentales: a) percepciones sobre la calidad del agua; b) problemas sociales, ambientales y culturales asociadas al deterioro de la calidad del agua; c) relación entre salud-trabajo y medio ambiente en los contextos locales. Este taller tuvo el objetivo de abordar las diversas percepciones frente a la relación entre salud-trabajo y medio ambiente, para propiciar una apropiación crítica de la realidad.

Por cada taller se conformaron grupos focales como herramienta que permite la construcción colectiva del conocimiento $(20,21)$. Se realizaron 13 grupos focales de entre 6 y 8 participantes por grupo, con líderes de Juntas de Acción Comunal
(JAC), Juntas de Acueductos Rurales (JAR), madres gestoras de la Red Unidos, y de la Secretaría Municipal de Salud. Adicionalmente, se realizaron cinco grupos con la población de niños y niñas de las escuelas rurales del sector.

El trabajo con los grupos focales buscó una descripción en extenso que permitiera comprender los conocimientos y acciones de los líderes organizados en colectivos. Se recolectó información en la totalidad de los territorios hasta llegar a la saturación, entendida como la no obtención de información nueva. Esta suficiencia de la información fue definida de común acuerdo por el grupo de investigación (22). Además, durante el trabajo con los grupos focales se identificaron actores claves de los territorios con los que se realizaron 36 entrevistas en profundidad, centradas en el tema del deterioro de la calidad del agua y en los procesos de salud-enfermedad. Para el registro de la información se utilizaron grabaciones en video y audio digitales, y cuadernos de anotaciones que posteriormente fueron transcritas en archivos de texto y llevadas para su codificación al programa informático Atlas.ti (versión 4.1). Se realizó análisis del discurso (23) producto de las transcripciones,

Cuadro 2. Guía de preguntas orientadoras diseñadas para el taller grupal. Municipio de Timbío, Departamento de Cauca, Colombia, entre noviembre de 2013 y agosto de 2014 .

1 ¿Qué percepción tienen de la calidad del agua que llega a sus hogares y/o que consumen?

2 ¿Cuáles son los principales problemas relacionados con el agua?; ¿cuáles son las actividades productivas que se desarrollan en su comunidad?; ¿cómo cree que afecta la calidad del agua?

3 ¿Cuáles son los principales problemas del manejo de aguas residuales y alcantarillado?

4 ¿Cuáles son las posibles fuentes de contaminación ambiental asociadas a las fuentes hídricas de abastecimiento?

$5 \quad$ ¿Cree usted que existe una relación entre las enfermedades y el agua que consumen?

6 ¿Qué actividades realizan para el cuidado del agua por parte de la comunidad y entes territoriales?

7 ¿Cuáles son las principales personas e instituciones y qué actividades ayudarían a resolver estos problemas?

8 ¿Qué acciones debería cambiar cada persona para resolver esta problemática?

Fuente: Elaboración propia. 
codificando los datos inicialmente con las categorías calidad del agua, percepciones sobre salud y fuentes de contaminación para cada territorio. Durante este análisis emergieron nuevas categorías como acciones de participación social o subcategorías como las relacionadas con los procesos de trabajo asociados a producción agroindustrial que deterioraban el agua, construyendo un marco de análisis representado en la Figura 1.

De igual manera, se realizó un taller utilizando la herramienta de cartografía social como una técnica dialógica, que invitó a la reflexión, organización y acción alrededor del territorio (24). Los líderes conocedores de la problemática del recurso hídrico en sus territorios identificaron las principales fuentes contaminantes mostradas en el mapa (Figura 2). Este reconocimiento de las interacciones de los colectivos entre el trabajo y el medio ambiente permitió la identificación de los estados de los sistemas de abastecimiento del recurso hídrico para la generación de mapas que fueron Ilevados a un Sistema de Información Geográfica (SIG) como se muestra en la Figura 2. El ejercicio permitió una mayor aproximación a la realidad y la construcción de un conocimiento integral del territorio, empleando herramientas técnicas en conjunto con los saberes populares.
Finalmente, en la fase denominada "acción", se presentó a los participantes una síntesis de la problematización desde variables cuantitativas (no incluidas en esta publicación) y cualitativas, y desde estos insumos se construyó una agenda de trabajo conjunta en el marco del proyecto y fuera de él. La centralidad de las acciones, que en la actualidad se están desarrollando en el marco del proyecto, están relacionadas con educación popular basada en el enfoque de derechos para la protección de la salud o el medio ambiente, en las que las organizaciones sociales ocuparon espacios políticos locales y generaron espacios de fortalecimiento organizativo en torno a la protección de la salud y el medio ambiente, con el acompañamiento técnico de los profesionales del proyecto.

\section{Procesamiento y análisis de la información}

El procesamiento de los datos se realizó a partir del análisis crítico y de la búsqueda de categorías fundamentales en los hechos descritos, indagando una comprensión en profundidad de los escenarios involucrados. Para el análisis de datos se realizaron lecturas en profundidad de las

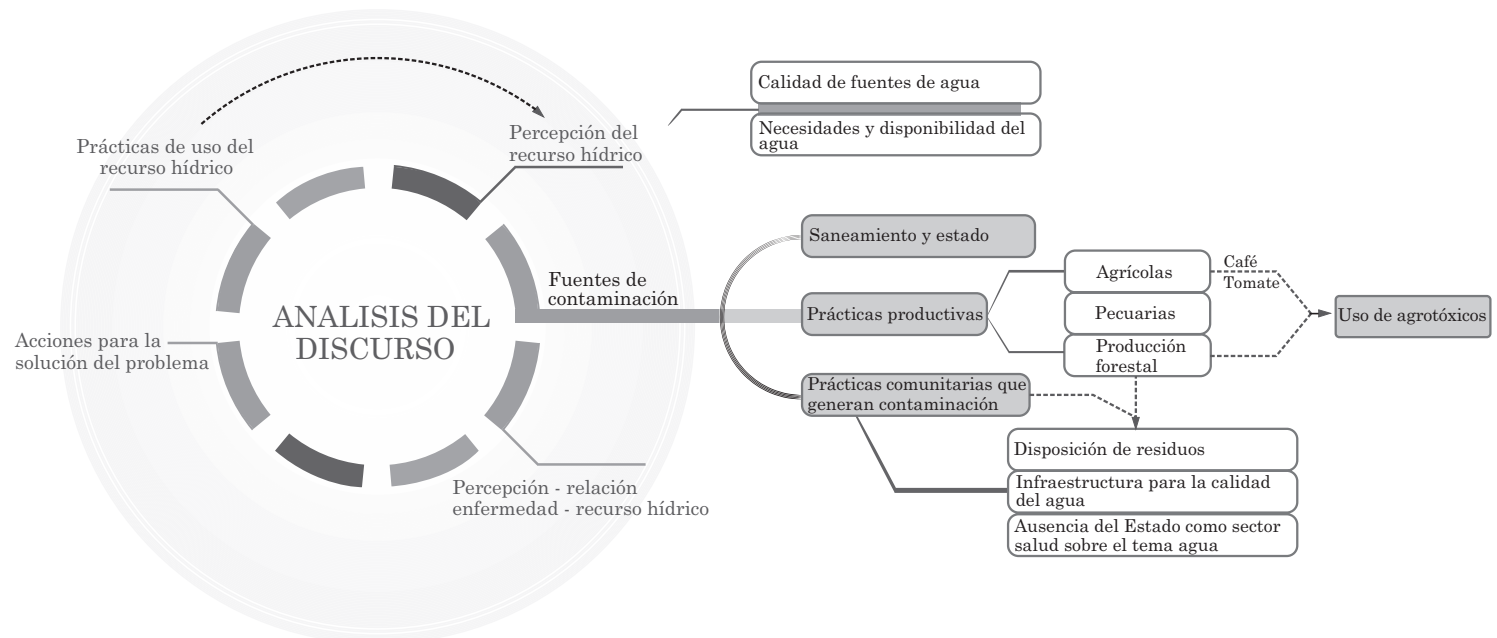

Figura 1. Mapa de análisis del discurso a partir de transcripciones de los relatos de la comunidad del municipio de Timbío, Departamento de Cauca, Colombia, entre noviembre de 2013 y agosto de 2014.

Fuente: Elaboración propia. 
transcripciones y discursos de la población y se contrastaron con los referentes teóricos de salud humana y aproximaciones críticas a la relación salud-medio ambiente-trabajo, buscando la construcción de un relato que evidencie, desde la articulación de los saberes científicos y populares, los procesos que favorecen el deterioro de los modos de vida, la salud, el trabajo y el medio ambiente.
Este estudio obtuvo el aval ético del Comité de Ética de la Universidad del Cauca, de conformidad con la Resolución No. 008430 de 1996, por la cual se establecen las normas científicas, técnicas y administrativas para la investigación en salud en Colombia.

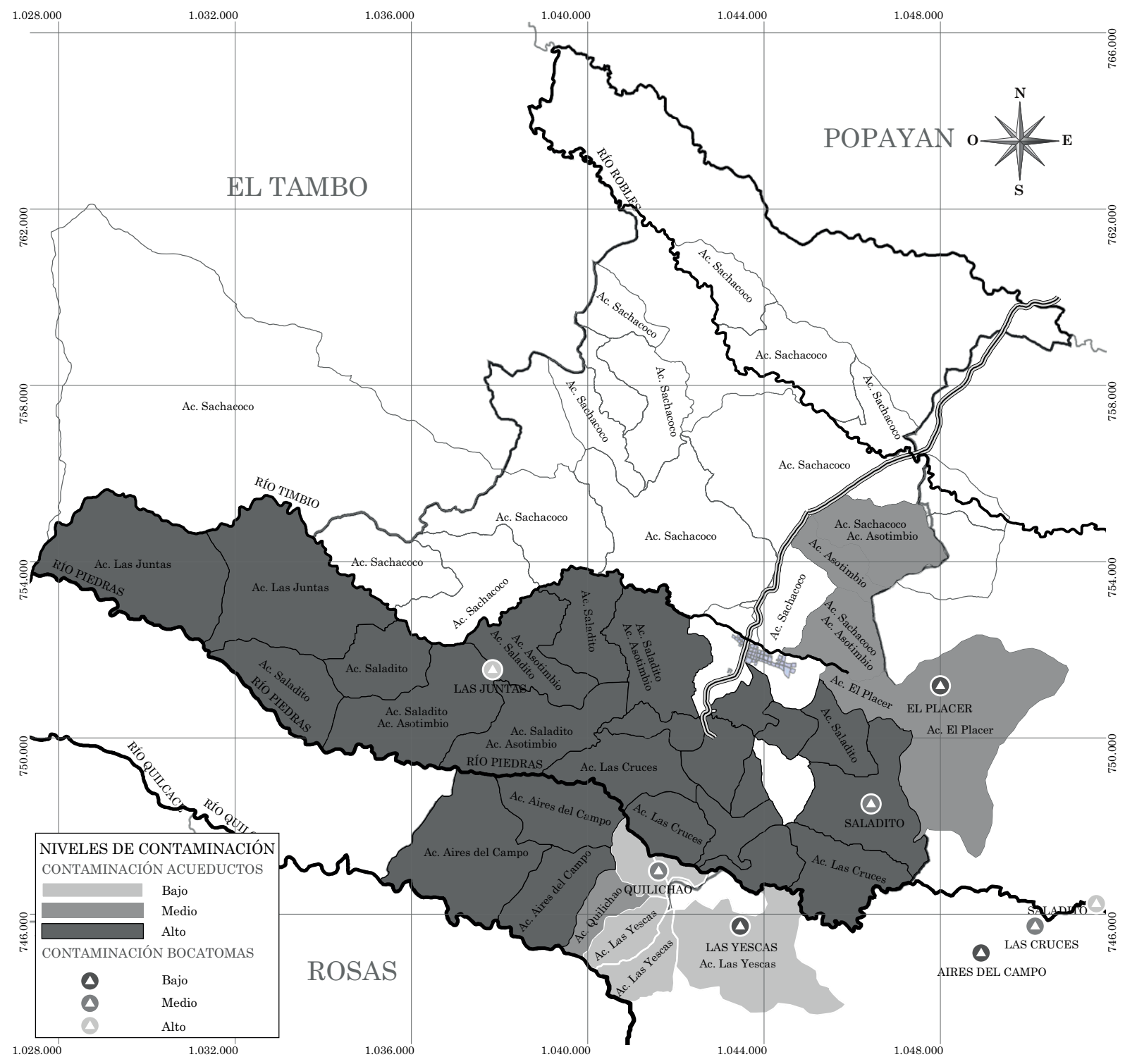

Figura 2. Mapa de riesgo elaborado a partir de la cartografía social, basado en las fuentes de contaminación pertenecientes a los acueductos rurales del municipio de Timbío, Departamento de Cauca, Colombia, entre noviembre de 2013 y agosto de 2014. 


\section{RESULTADOS Y DISCUSIÓN}

La aproximación ecosistémica de los problemas ambientales y de salud humana busca definir estrategias para mejorar su control. Esto exige una reconciliación entre el hombre y la naturaleza que favorezca el mejoramiento de la salud colectiva, una conexión entre las actividades de las personas - propias de sus culturas- y el impacto en el entorno, entre el estado de los ecosistemas y la salud de las personas.

Si bien este trabajo no pretende reconstruir la relación hombre-naturaleza, dado que implicaría una mayor profundización, sí pretende reivindicar, como método, la transdisciplinariedad, haber rescatado el saber popular para la construcción del conocimiento a partir del diálogo de saberes (saber científico y saber popular) en lugar de enmascarar sus respuestas en nuestras propias categorías. Partimos de la comprensión social de la visión de los actores sociales, desde una perspectiva interpretativa, intentando codificar sus visiones y acciones; es decir, generar cambios a partir de evidencias construidas participativamente, entendiendo que la cooperación de los individuos conecta la investigación con la acción.

Para el análisis de las percepciones de los grupos sociales, se elaboró un sistema de codificación de información cualitativa. La percepción de la calidad del agua en las comunidades suele asociarse, en sus discursos, con el olor y el aspecto $y$, consecuentemente, está estrechamente relacionada con las fuentes de contaminación y la falta de plantas de tratamiento para potabilizar el agua en todos los acueductos rurales. Por consiguiente, las comunidades buscan con esfuerzo acciones para potabilizar el agua de consumo, tal como lo mencionan resultados similares encontrados en otras investigaciones que abordan las percepciones locales en relación con el agua, sus ambientes y usos $(25,26)$. En general, la comunidad percibe una mala calidad del agua de consumo: "...el agua cuando usted abre la llave llega con mucha mugre", mientras otra parte de la comunidad considera que el agua es mala porque se enferman. Los líderes indican, además, que el agua es mala porque "...en épocas de lluvia baja muy oscura y con pelos" y, por tal motivo, deciden tomarla de fuentes alternas como quebradas, ríos o nacimientos. Estudios explicativos similares muestran que las percepciones de la calidad del agua en las comunidades podrían estar condicionando la elección de las diferentes fuentes para su uso y, por lo tanto, algunas comunidades prefieren el consumo de agua de aljibes, pozos familiares, entre otros (26).

\section{Procesos de producción rural y su relación con el deterioro del agua}

A medida que la interacción hombre-naturaleza se ha intensificado, han avanzado los deterioros de esta relación y la generación de ambientes no saludables. Un claro ejemplo es la evidencia histórica del uso y manejo del agua, asociado a las actividades íntimamente ligadas a la producción agropecuaria, eje y sostén socioeconómicos regionales (27). Durante este análisis, uno de los elementos emergentes en la transformación del medio ambiente -especialmente en el deterioro y disponibilidad del agua- corresponde a los procesos productivos relacionados con la categoría de trabajo en el territorio. Dentro de estos se encuentra la producción agroindustrial, especialmente en maderables, identificada claramente por mujeres y hombres:

\footnotetext{
...le están haciendo mucho daño al agua porque ellos están haciendo plantaciones muy cerca del agua y eso pues hace que los árboles consuman mucha agua y el agua se está mermando mucho... (Mujer adulta, líder del acueducto El Saladito)
}

Uno de los principales conflictos sobre el manejo y uso del agua en Timbío aparece por los impactos en la disponibilidad de agua, dado que este recurso se ve afectado por la producción de maderables. Sin embargo, no es solo la percepción de las poblaciones. Diversos estudios hidrológicos realizados en otras regiones del mundo han encontrado impactos sobre la disponibilidad del agua en regiones con cultivos agroindustriales de eucalipto y pino, como los encontrados en Timbío (28). La percepción es que los procesos productivos en sí mismos deterioran las fuentes de agua y su disponibilidad en los acueductos. En este proceso, se destaca el uso de maquinarias pesadas para la transformación de la madera: 
...por lo menos está bajando el agua sucia porque allá hay mucha maquinaria, sacando madera, entonces cuando va pasando por la cañada, bate ese barro y por eso es que baja sucia... (Hombre adulto, líder del acueducto El Saladito)

La incorporación de los saberes comunitarios y la subjetividad, desde el reconocimiento de sus conocimientos, creencias y modos de vida en relación con la utilización de agrotóxicos, ha permitido, en otros estudios, problematizar la complejidad de los procesos socio-históricos que determinan la relación salud-trabajo. Esta construcción desde el diálogo de diferentes saberes genera nuevas estrategias para garantizar la salud y seguridad en el trabajo de las poblaciones (29).

El uso de agrotóxicos necesarios para el control de plagas en monocultivos forma parte de las percepciones sobre el deterioro del agua:

\footnotetext{
...el agua que nos estamos tomando, sospechamos que es contaminada [...] lo otro que supremamente grave y delicado es que estamos rodeados de plantas de Cartón Colombia que permanentemente son tratadas con agroquímicos que nos están vertiendo esos residuos contaminantes a nuestra fuente de agua. (Hombre adulto, líder de la vereda Barro Blanco).
}

El imaginario popular y del mundo en general ha visibilizado la relación destructiva derivada de la utilización de plaguicidas en las comunidades locales, gracias a la aparición de investigaciones científicas que indican el deterioro en la salud vinculado a los agrotóxicos, especialmente al uso de los agrotóxicos como el glifosato (30). Aunque se han realizado estudios e informes sobre los efectos adversos de los agrotóxicos, tanto en las personas como en el medio ambiente, no se ha documentado en profundidad cuáles son las consecuencias de dichos daños en las comunidades rurales y cómo estas se ven afectadas en el uso y manejo del agua como recurso natural:

El eucalipto por ser árboles tan grandes esos los colocan en toda la orilla del agua, entonces ahí llegan las fumigaciones y caen a la propia quebrada que va a surtir el acueducto
[...] Es que toda la maleza la fumigan con glifosato y eso es peligroso. (Mujer adulta, líder del acueducto El Saladito)

\section{Uso de agrotóxicos}

...hay cultivos de fresa que dejan plástico, tarros de venenos que uno encuentra en las "cañadas". (Hombre adulto, fontanero del acueducto, vereda Camposano)

\begin{abstract}
...la ganadería pues hace uso casi mensual de "garrapaticidas" que si sumamos de frasco en frasco eso va a hacer una cantidad... (Hombre adulto, líder de la vereda Cristaleres)
\end{abstract}

Desde los años sesenta se ha desarrollado este modelo de producción agroindustrial que, según reporta el plan de manejo ambiental, alcanza los $169 \mathrm{~km}$ de monocultivos de especies forestales en el municipio. Sin embargo, no solo los procesos productivos agroindustriales maderables, sino también la cría de especies pecuarias y pequeños cultivos aparecen como elementos de deterioro de la calidad del agua. El manejo de excretas por parte de los trabajadores de la empresa maderera contamina con heces fecales la parte alta de las cuencas de donde posteriormente se tomará el agua para los acueductos:

\begin{abstract}
A pesar de letrinas, no todos los trabajadores las utilizan, lo que lleva a que tengamos contaminación por heces fecales en determinados tiempos, por eso cada vez que hay cosecha los focos de contaminación son grandes. (Hombre adulto, líder del distrito de riego Asotimbio)
\end{abstract}

\section{Contaminación de fuentes hídricas por heces de especies pecuarias}

Las regiones rurales de Colombia tienen una población del 32\% calculada por el Programa de las Naciones Unidas para el Desarrollo (PNUD). Esta fuente indica, además, que el $75 \%$ de los municipios del país son más rurales que urbanos y, en el caso del municipio de Timbío, la población rural es mayoritaria (31). Estas poblaciones en su 
mayoría realizan actividades agropecuarias como propietarios o como mano de obra de grandes procesos agroindustriales. Las formas de producción en el municipio se ven influenciadas por diversas políticas públicas basadas en los principios fundamentales del mercado, que determina las relaciones sociales y la competencia entre los sujetos como dinamizador del progreso, que como expresión técnica y de trabajo rural se materializa en una producción de monocultivos a pequeña y gran escala, que necesitan de tecnologías como la mecanización y de agrotóxicos para la obtención de productos agrícolas (32).

Estas lógicas de desestructuración de las formas de producción campesina tradicional en la región han favorecido la concentración de riquezas y el deterioro ambiental (33). Las estructuras de producción rural han generado un desequilibrio de los agroecosistemas, que deterioran la calidad del agua por múltiples fuentes de contaminación (agrotóxicos, maquinarias agrícolas, desechos del proceso productivo y humanos) $(34,35)$. Las comunidades de Timbío conviven con empresas multinacionales y diversos pequeños productores agropecuarios que deterioran sus condiciones ecológicas y determinan procesos de concentración de los recursos ambientales, particularmente, el agua, lo cual produce un deterioro de los procesos de salud-enfermedad del colectivo (36). En el análisis planteado para esta investigación, la relación entre el trabajo y el medio ambiente fue fundamental, dado que la categoría trabajo es el acto conciente realizado por los colectivos humanos para transformar el medio ambiente, obtener en este caso productos agrícolas propios del sustento y que, a su vez, transforma las condiciones humanas de vida y salud. Esta relación involucra profundamente al medio ambiente con el aparato productivo de la región, ya que ofrece los medios de producción, las materias primas para ser transformadas y las condiciones ambientales para la reproducción social de la fuerza de trabajo, elementos fundamentales para el desarrollo y la salud humana (37).

...los animales que están pastando van a beber el agua al río, mas en la parte hacia abajo, donde canalizamos ya el agua para el acueducto, entonces se dice que son estos animales los que nos contaminan el agua. (Hombre adulto, líder de la vereda San Pedrito)

\begin{abstract}
...marraneras y pues me contaba un vecino que han hablado con los de sanidad, pero que solamente vienen a mirar y que no le ponen mano dura a eso, y cuando uno pasa eso huele horrible. (Hombre adulto, líder del acueducto El Saladito)
\end{abstract}

\section{Estado y protección del medio ambiente y la salud}

La generación de conflictos socioambientales, por la interrelación de actores en una sociedad, en áreas urbanas o rurales, involucra directamente al Estado como responsable del bienestar de los diferentes sectores de la sociedad. La pugna con los gobiernos centrales por el derecho al agua se manifiesta cuando entran agentes externos, amparados por el Estado, para la extracción de recursos naturales y otras actividades al interior de las zonas donde se encuentra el recurso natural, a pesar de las normativas (que parten con la Constitución). Mediante la Ley 99 de 1993, se creó el Ministerio del Medio Ambiente y se organizó el Sistema Nacional Ambiental (SINA). En particular sobre el agua, en el Artículo $1^{\circ}$, la ley establece que "las zonas de páramos, subpáramos, los nacimientos de agua y las zonas de recarga de acuíferos serán objeto de protección especial", y que "en la utilización de los recursos hídricos, el consumo humano tendrá prioridad sobre cualquier otro uso" (38). En Colombia, el Consejo Nacional de Política Económica y Social, a través del documento Conpes 3343 “Lineamientos y estrategias de desarrollo sostenible para los sectores de agua, ambiente y desarrollo territorial" (39), con relación al agua, explicita las temáticas que debería incluir la Ley de Agua: el derecho al uso del agua, estándares de calidad, control de contaminación (40). Sin embargo, existe una ausencia del Estado frente a la gestión del recurso hídrico en la ruralidad, percibida por las comunidades.

Los procesos de deterioro ambiental determinados por los procesos productivos, y por ende de la salud de los colectivos de Timbío, se desarrollan en parte, por la desaparición del Estado en la protección de las fuentes hídricas, la potabilización del agua y en su acción de control frente al saneamiento básico y la prevención del deterioro ambiental: 
...es el Estado, decimos que tenemos unos derechos pero estos son vulnerados porque no nos brindan ese derecho, hay entidades las cuales deben vigilar por la calidad del medio ambiente y porque no haya más contaminación, y cuando nosotros como asociación solicitamos una ayuda, lo que hacen es ponernos a hacer un poco de trámites, sin embargo, cuando llega una multinacional pasa todo lo contrario. (Hombre adulto, líder de la vereda Buenos Aires)

Esta percepción de la protección del derecho al medio ambiente sano por parte del Estado se expresa en diferentes instituciones:

Pues se dice que el Ministerio de Medio Ambiente es el que debe estar pendiente de todo eso, en cabeza del Estado. (Hombre adulto, líder del acueducto rural El Placer)

...la CRC, pues siempre se ha escuchado que son ellos los que tienen que estar pendiente del medio ambiente y todo lo relacionado con eso, por eso pienso que esa entidad es la más adecuada. (Hombre adulto, líder del acueducto rural El Placer)

\footnotetext{
...la gente tiene que saber que política es una cosa y politiquería es otra cosa. Existe lo que se llama política de desarrollo y eso es hacer las cosas bien para que podamos producir y no hagamos daño, digamos, al medio ambiente. Entonces yo entiendo que política son las decisiones que tomemos, pero muchos creen que política es esa politiquería que hacen los políticos tradicionales y resulta que ellos lo que hacen es aprovechar un voto para hacer lo que tienen que hacer con el tesoro público, entonces lo que nosotros tenemos que hacer es resolver las cosas y exigir al Estado para que se resuelva de raíz los problemas... (Hombre adulto líder del acueducto El Saladito)
}

El deterioro del recurso hídrico, además, se ve afectado por factores como la disposición inadecuada de residuos y los vertimientos y asentamientos. El único método utilizado en el municipio de Timbío para la disposición final de los residuos sólidos es el basural a cielo abierto, Ilamado por las comunidades "relleno sanitario". Es un espacio destinado para la disposición y la degradación de los residuos tanto de la zona urbana como de la zona rural, proceso en el que se generan varios tipos de emisiones contaminantes. Debido a la generación de dichos contaminantes, este tipo de espacios deben estar ubicados en zonas alejadas del espacio habitacional y de las nacientes de agua utilizadas para el consumo humano; sin embargo, en el municipio de Timbío se encuentran a 700 metros de una de las bocatomas del acueducto "El Saladito". La contaminación hídrica podría darse por lixiviados generados por la descomposición de la materia orgánica o animales vectores como los perros o las aves de rapiña. Según el Plan de Salud Territorial del municipio, las enfermedades generadas por mala disposición de residuos sólidos han generado impacto en la salud pública en la región (7).

La contaminación de las fuentes hídricas generada por el relleno sanitario es una condición claramente problematizada por los líderes de los acueductos veredales:

...bueno, el relleno sanitario queda como a unos 800 metros pero, yo creo que cuando hicieron el rellenos sanitario eso lo avalaron porque pues siempre hay desnivel, entonces a nosotros nos afecta mucho porque la bocata queda hacia arriba y el relleno queda hacia abajo, eso ya hace muchos años que eso está funcionando, pero las estadísticas no nos dicen que la muerte se haya enfermado por tomar esa agua. (Hombre adulto, líder del acueducto El Saladito)

...en muy malas condiciones ahora en este periodo con esta alcaldesa, era un desastre a cielo abierto y todo, y lo que son las aves de carroña, como ahí llega todo lo que es hueso de carnicería y ellos llegan a comer, y estos vuelan a bañarse a la quebrada y eso es lo que lo contamina. (Hombre adulto, líder de acueducto El Saladito)

\section{Ausencia de plantas de potabilización}

Los procesos de deterioro ambiental y la desaparición del Estado en la protección del medio 
ambiente se demuestra en las bajas coberturas de agua potable y saneamiento básico. Si bien el $56 \%$ de la población rural en Colombia tiene acceso a alguna forma de abastecimiento de agua, solo el $12 \%$ cuenta con agua tratada. En relación con el servicio de saneamiento, tan solo el $34 \%$ de los habitantes rurales cuenta con algún sistema de evacuación de aguas servidas, el 29\% con soluciones individuales en sitio, tales como letrinas y tanques sépticos, y el 5\% utiliza alcantarillados convencionales; el $66 \%$ restante no contaba con ningún tipo de sistema (41-43).

La situación del Cauca es similar, con un servicio de alcantarillado que no llega ni siquiera a la mitad de los hogares en el área rural y la cobertura del servicio de acueducto en las cabeceras municipales es apenas del $66 \%$ :

El acueducto, por ejemplo, la construcción debió haber sido por parte del Estado, y no por la comunidad que fue como sucedió, por eso el Estado tiene la obligación de educar a la gente, de hacer que haya campañas culturales para que no cometamos errores sobre todo en el campo con el tratamiento de las aguas. (Hombre adulto, líder vereda Cristalares)

...el gobierno como la alcaldía deben encargarse de que la planta de tratamiento se haga y también la comunidad es responsable porque se debe cuidar los nacederos y no contaminar más el agua. (Hombre adulto, líder vereda Cristalares)

\section{Garantía del derecho a la salud}

El derecho a la salud no solo se garantiza con la atención oportuna y apropiada de los eventos asociados a la contaminación del agua. El derecho a la salud abordado integralmente incorpora elementos fundamentales, tales como el acceso a alimentos sanos y nutrición adecuada, vivienda adecuada, agua limpia y potable, condiciones sanitarias adecuadas y un medio ambiente sano (44). En esta lógica, la reaparición del Estado en la recuperación y promoción del cuidado del agua debe ser una prioridad, en la que se vinculan las acciones colectivas como mecanismo de exigibilidad del derecho.
En América Latina, estados clientelistas en los que el ejercicio de los derechos sociales está en parte supeditado a los favores políticos, se evidencia el debilitamiento y la carencia de procesos de vigilancia, control y veeduría por parte del gobierno y los ciudadanos (45). En estas circunstancias, los más afectados son los segmentos más vulnerables de la población. La protección ambiental y la reducción de los efectos nocivos del ambiente en la salud se han convertido en requisitos inseparables de los esfuerzos para construir un proceso efectivo y sostenido de desarrollo económico y social (46). Sin embargo, no se avanza mucho en el diseño, la organización y la ejecución de acciones tendientes a impedir o a revertir los efectos nocivos del ambiente sobre la salud humana, especialmente en la ruralidad:

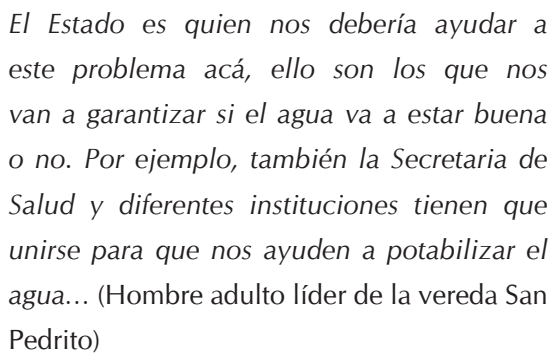

El conocimiento acerca de la salud es un elemento esencial en muchos programas de alfabetización y educación básica. Sin embargo, muchos de estos programas se concentran en la mujer e incluyen nutrición, higiene y planificación familiar. La educación para la salud frecuentemente se combina con otras medidas para mejorar el bienestar y fomentar el desarrollo de la comunidad (47). El acceso a educación e información en salud y la participación de la población en todo el proceso de adopción de decisiones es uno de los factores que emerge desde los colectivos:

\footnotetext{
A mí me gustaría que se hiciera una brigada de salud, para mirar en qué grado de contaminación está la población. [...] yo pienso que la acción de tratamiento del agua, desde que todos estamos aquí reunidos, pues ojala salir con una capacitación que nos den ustedes que vienen y agradecemos por estar aquí, y que ojala para todos los niños, porque nosotros ya estamos viejos, nuestros niños,
} 
nuestros nietos, y queremos de que el agua sea tratada y pues aquí, por una parte, de que uno lo llevan al hospital hay mala atención y que ojala el doctor que está aquí nos ayudara en ese sentido. (Hombre adulto, líder de la vereda Cristalares)

...hasta que nosotros decidamos, para cambiar la situación no solo depende de la alcaldesa, depende de la gestión que nosotros podemos hacer, si nosotros con este podemos generar por ejemplo políticas y presentamos resultados y generar políticas de mejoramiento del ambiente o la calidad del agua, entonces eso es lo que tenemos que lograr [...] Necesitamos mostrar evidencias, vamos a hacer análisis de calidad del agua, análisis en algunos de ustedes para ver si tienen alguna enfermedad y con toda esa información y con todos ustedes, ahora sí, hacer gestión. (Hombre adulto, líder de la vereda Cristalares)

\section{Agua, salud y enfermedad}

En cuanto a los estados de salud y enfermedad, en el año 2011, el municipio de Timbío reportó grandes problemas de salud pública como las enfermedades trasmitidas por alimentos (ETA) tanto en niños como en adultos mayores. Según los datos estadísticos encontrados y reportados por el Centro de Salud de Timbío, entre las diez primeras causas de morbilidad está la parasitosis intestinal, sin otra especificación, la diarrea y gastroenteritis de presunto origen infeccioso y la infección aguda de las vías respiratorias superiores, encontrándose la neumonía no especificada como la primera causa con 96 casos. Estas causas podrían estar relacionadas con el posible papel del agua como reservorio natural de patógenos emergentes como Helicobacter pylori, agente etiológico del cáncer de estómago, siendo la primera causa de muerte por cáncer en esta población (48).

En los talleres comunitarios, los líderes reconocen claramente la relación existente entre la presencia de alteraciones en la salud como diarreas, alergias, brotes en la piel y la mala calidad del agua que perciben:

\begin{abstract}
...allá habitan mucha gente adulta y por lo menos los niños viven muy enfermos o desnutridos, pero es por la cuestión del agua... (Mujer adulta, líder de la vereda San Pedrito)
\end{abstract}

Siempre llega el agua sucia, con tierra, a veces baja con mal olor y sabor... lo expresan aterrados de esta situación, otras veces baja con gusanitos y lombrices... no es recomendable para consumirlo, sin hervirla o tratarla con algún químico... (Mujer adulta, líder de la vereda San Pedrito)

...también hemos visto cadáveres de animales y las otras veces sacaron un muerto del río Pambío. Siempre lavan los fumigadores en las fuentes de agua... (Mujer adulta líder de la vereda San Pedrito)

Es claro que la ruta fecal-oral es bastante plausible para la transmisión de Helicobacter pylori, en la que el agua juega un rol importante como vector. Sin embargo, las comunidades no identifican la relación entre el agua contaminada con heces y su posible transmisión de ese patógeno. Este aspecto genera inquietudes entre los líderes, quienes se preguntan acerca del papel del agua en el proceso de transmisión y desarrollan creencias o "teorías intuitivas" (49). Su conocimiento se aproxima a la visión médica y lo relacionan con alteraciones gástricas, incluso con el cáncer de estomago. Sin embargo, identifican la ausencia del sector salud frente a los programas educativos al respecto. Según las creencias populares hay dos conceptos respecto a las causas de enfermedad en las comunidades: una indica que la enfermedad tiene origen en el agua, la otra señala que es causada directamente por los alimentos contaminados. Ambos conceptos reconocen que la génesis de las enfermedades gástricas está en el agua; lo que genera cuestionamientos entre los líderes acerca del papel de Helicobacter pylori y su transmisión, queriendo dar respuesta a aquello que no pueden explicar. En esta construcción de teorías populares sobre la relación entre los estados de salud-enfermedad y la naturaleza "agua", se fusionan además saberes tradicionales y saberes científicos: 
...el cáncer gástrico es una de las enfermedades más comunes en nuestro municipio. En mi familia, hay muy buena parte de la familia Muñoz que ha fallecido por cáncer gástrico, y eso es ahí en la vereda el Hato, en alguna ocasión se presentó allá unos problemas gástricos en los niños pero para mí el problema ha sido el consumo del agua contaminada... (Hombre adulto, líder comunitario vereda La Alfonsa)

...mi hija tiene 11 años, una vez se enfermó de un dolor fuerte en el estómago, entonces la lleve al hospital porque tenía el estómago hinchado, quedó hospitalizada, pero en Popayán no había un gastropediatra, entonces me la remitieron a Cali, allá le hicieron una endoscopía y entonces le detectaron la Helicobacter pylori, le diagnosticaron gastritis crónica, le enviaron unos medicamentos, yo se los di y eso me la controló por un tiempo. Ella se enfermó no solo por consumir esa agua contaminada, sino también por los alimentos, porque esos alimentos también son tratados con muchos químicos, además en nuestra comunidad también se han escuchado de enfermedades como la diarrea y enfermedades parasíticas. (Madre comunitaria, vereda La Alfonsa)

El análisis de la compleja matriz de una problemática de salud como la hídrica, y la correlación metodológica operada a través del enfoque transdisciplinar, ponen en evidencia el carácter ecosistémico que atraviesa este trabajo de salud ambiental. Además, se expone un abordaje de salud intercultural y se pone de manifiesto la necesidad de trabajar en acciones de prevención y promoción de la salud que permitan un uso apropiado de las fuentes de agua; la inclusión de los saberes; la formación de agentes sanitarios y promotores de salud; el desarrollo de estrategias con la comunidad como alternativas para mejorar la calidad del agua (hervir el agua, aplicar cloro, construir filtros caseros); y la participación en espacios políticos que permitan la reivindicación de derechos a la salud y el agua limpia.

\section{REFLEXIONES FINALES}

La interrelación de los investigadores, la comunidad y los representantes de los sectores en el análisis conjunto durante el desarrollo de las etapas del proyecto propició la construcción de las interfaces entre varias disciplinas complementarias, fortaleció la integración del conocimiento y la adopción de un lenguaje común. Este proceso tuvo un efecto sinérgico en su transformación hacia un equipo transdisciplinario. Además, es claro que la participación comunitaria se convierte en un espacio de incertidumbre, pero también en un espacio para bordar la realidad de forma integral, accediendo al intercambio de conocimientos y al análisis conjunto de los problemas que afectan a las comunidades. Este enfoque permitió la identificación de acciones de transformación en los contextos sociales, ecológicos y políticos. Se identificaron responsables de la problemática relacionada con el agua, acciones para controlar la problemática y el tiempo necesario para ejecutarlas.

Durante el desarrollo de esta etapa se fortaleció la integración del conocimiento y la adopción de un lenguaje común, aunque se mostró que el conocimiento no genera por sí mismo cambios de actitud, ni de comportamiento, pero sí abona el terreno para cambiar acciones y retroalimentar los saberes. Se evidenciaron procesos de interacción, discusión y elaboración de acuerdos que determinaron los conocimientos, actitudes y creencias relacionados con los estados de salud, la presencia de fuentes de contaminación asociadas a las principales fuentes de agua para el consumo, condiciones de saneamiento, así como las características de las diferentes zonas geográficas.

Se activaron espacios políticos en busca de estrategias de acompañamiento, desde la institucionalidad y desde un marco político. Esto pone de manifiesto que la práctica de investigar en forma participativa también puede ser entendida como un fenómeno político en el que los actores sociales tienen la posibilidad de transformar su cotidianidad y de vincularse políticamente con el Estado, buscando disputar el poder para promover la protección de derechos, mejorar sus condiciones de vida y evitar el deterioro del medio ambiente y los efectos sobre la salud en el contexto del actual modelo de producción. 
La perspectiva de cartografía social permitió reconocer e incorporar en la investigación los intereses y las posiciones también políticas de la comunidad y de las organizaciones sociales, las cuales se expresaron en las indicaciones específicas que los participantes trazaron sobre los mapas. Este ejercicio de cartografía se enmarcó en la reflexión y definición de acciones antrópicas relacionadas con la contaminación del agua, que permitieron además visibilizar las disputas por la presencia de organizaciones privadas con intereses en la producción agrícola, en las cuales las comunidades perciben emisiones de contaminación en las fuentes de agua, con pocas acciones concretas de mitigación y/o prevención.

\section{AGRADECIMIENTOS}

A las comunidades locales del municipio de Timbío por su participación en la investigación, aportando sus conocimientos con disposición. A la Vicerrectoría de Investigaciones de la Universidad del Cauca, a la Fundación InnovaGen y a la Universidad EAN por el apoyo en la ejecución de esta investigación. Este estudio fue financiado por el Programa Nacional de Ciencia y Tecnología de la Salud de Colciencias, Código 110356934947.

\section{REFERENCIAS BIBLIOGRAFICAS}

1. Parkes M, Horwitz P. Water, ecology and health: ecosystems as settings for promoting health and sustainability. Health Promotion International. 2009;24(1):94-102.

2. Álvarez A, García A, Bonet M. Pautas conceptuales y metodológicas para explicar los determinantes de los niveles de salud en Cuba. Revista Cubana de Salud Pública. 2007;33(2).

3. Organización Mundial de la Salud. Agua para la salud: un derecho humano [Internet]. Ginebra: Organización Mundial de la Salud; 27 nov 2002 [citado 19 dic 2011]. Disponible en: http://goo. gl/9MjeNx.

4. Prüss-Üstün A, Bonjour $\mathrm{S}$, Corvalán C. The impact of the environment on health by country: a meta-synthesis. Environmental Health. 2008;7(7):1-10.

5. Consejo Nacional de Política Económica y Social. Lineamientos para la formulación de la política integral de salud ambiental con énfasis en los componentes de calidad de aire, calidad de agua y seguridad química (Documento Conpes 3550). Bogotá: Ministerio de Salud y Protección Social; 2008.

6. Soto-Moreno JA, Ballester-Díez F. Contaminación del aire de interiores en hogares en situación de pobreza extrema en Colombia. Revista de Salud Pública. 2013;15(1):80-89.
7. Alcaldía Municipio de Timbío. Plan Territorial de Salud 2008 [Internet] 2008 [citado 24 nov 2011]. Disponible en: http://goo.gl/qT0bVc.

8. Adrada JC, Calambás FH, Díaz JE, Delgado DO, Sierra $\mathrm{CH}$. Características sociodemográficas y clínicas en una población con cáncer gástrico en el Cauca, Colombia. Revista Colombiana de Gastroenterología. 2008;23(4):309-314.

9. Charron DF. Ecosystem approaches to health for a global sustainability agenda. EcoHealth. 2012;9(3):256-266.

10. Basso C. Abordaje ecosistémico para prevenir y controlar al vector del dengue en Uruguay. Montevideo: Universidad de la República; 2010.

11. Betancourt $O$, Barriga $R$, Guimarães JRD, Cueva E, Betancourt S. Impacts on environmental health of small-scale gold mining in Ecuador. In: Charron DF, editor. Ecohealth Research in Practice: Innovative Applications of an Ecosystem Approach to Health. New York: Springer; 2012. p. 119-130.

12. Lebel J. Salud: un enfoque ecosistémico. Bogotá: Centro Internacional de Investigaciones para el Desarrollo, Alfaomega Colombiana; 2005.

13. Dakubo CY. Ecosystems and human health: a critical approach to ecohealth research and practice. New York: Springer Science \& Business Media; 2010. 
14. Mertens F, Saint-Charles J, Mergler D. Social communication network analysis of the role of participatory research in the adoption of new fish consumption behaviors. Social Sciences \& Medicine. 2012;75(4):643-650.

15. Ortiz M, Borjas B. La investigación acción participativa: aporte de Fals Borda a la educación popular. Espacio Abierto. 2008;17(4):615-627.

16. Fals-Borda O. Antología. Bogotá: Editorial Universidad Nacional de Colombia; 2010.

17. Chevalier J, Buckles D. Guía para la investigación colaborativa y la movilización social (SAS2). México DF: Plaza y Valdés, Centro Internacional de Investigaciones para el Desarrollo; 2009.

18. Leal E. La investigación acción participación, un aporte al conocimiento y a la transformación de Latinoamérica, en permanente movimiento. Revista de Investigación. 2009;(67):13-34.

19. González Rey F. Investigación cualitativa y subjetividad: Los procesos de construcción de la información. México DF: MacGraw-Hill; 2007.

20. Vallejo M, Martínez JD. Tarea y competencia comunicativas: reflexiones desde un grupo focal. Íkala. 2011;16(28):161-197.

21. Tomat C. El 'focus group': nuevo potencial de aplicación en el estudio de la acústica urbana. Athenea Digital. 2012;12(2):129-152.

22. Martínez-Salgado C. El muestreo en investigación cualitativa: Principios básicos y algunas controversias. Ciência \& Saúde Coletiva. 2012;17(3):613-619.

23. Minayo MCS. La artesanía de la investigación cualitativa. Buenos Aires: Lugar Editorial; 2009.

24. Borda OF. Participatory (action) research in social theory: Origins and challenges. In: Reason $\mathrm{P}$, Bradbury $\mathrm{H}$. Handbook of action research: Participative inquiry and practice. London: SAGE; 2001. p. 27-37.

25. Dolnicar S, Schäfer Al. Desalinated versus recycled water: public perceptions and profiles of the accepters. Journal of Environmental Management. 2009;90(2):888-900.

26. Martínez GJ, Beccaglia AM, Llinares A. Problemática hídrico-sanitaria, percepción local y calidad de fuentes de agua en una comunidad toba (qom) del Impenetrable (Chaco, Argentina). Salud Colectiva. 2014;10(2):225-242.
27. Montico S. El manejo del agua en el sector rural de la región pampeana argentina. Theomai. 2004;(1):7.

28. Hofstede R, Lips J, Jongsma W. Geografía, ecología y forestación de la Sierra Alta del Ecuador: Revisión de literatura. Quito: Abya-Yala; 1998.

29. Piracón JAB, Lozada MA. Prácticas de fumigación en cultivadores de papa, saberes y creencias sobre los plaguicidas y la salud. Tempus Actas de Saúde Coletiva. 2014;8(2):93-108.

30. Castro FG, Agudelo SMG, Vera MEB. Efecto de la aplicación de glifosato en la flora y microflora asociada a arvenses en el cultivo de café (Coffea arabiga L) en el municipio de Santa Rosa de Cabal, departamento de Risaralda. Cultura y Droga. 2010;8(10):225-254.

31. Bruno M. Hechos de Paz: La hora de la Colombia rural. Bogotá: PNUD; 2012.

32. Breilh J. Aceleración agroindustrial: peligros de la nueva ruralidad del capital. En: Brassel F, Breilh J, Zapatta A. ¿Agroindustria y soberanía alimentaria?: hacia una ley de agroindustria y empleo agrícola. Quito: SIPAE; 2011. p. 171-190.

33. Breilh J. Despojo, ecosistemas y salud. Ponencia presentada en: III Seminario Nacional sobre Ambiente y Salud. 20-22 sep 2004; Rio de Janeiro, Brasil: Fundação Oswaldo Cruz.

34. Neumann F. Cultivo de la papa. México: Iberoamericana; 2001.

35. Henríquez G. Panorama de los conflictos ambientales en la región del Biobio. Santiago: Observatorio Latinoamericano de Conflictos Ambientales; 2003.

36. Lozada M, Muñoz A. Experiencias de investigación en salud y seguridad en el trabajo. Bogotá: Universidad Nacional de Colombia; 2012.

37. Rothman H. La barbarie ecológica: estudio sobre la polución en la sociedad industrial. Barcelona: Fontamara; 1980.

38. Colombia. Ley 99 de 1993 [Internet] 22 dic 1993 [citado 15 sep 2014]. Disponible en: http:// goo.gl/O4zDQb.

39. Consejo Nacional de Política Económica y Social. Lineamientos y estrategias de desarrollo sostenible para los sectores de agua, ambiente y desarrollo territorial (Documento Conpes 3343) [Internet]. 2005 [citado 15 ago 2014]. Disponible en: https://goo.gl/MKCCx5. 
40. Quiñonez Rizo EA. Gerencia ambiental en Colombia. Ambiente y Desarrollo. 2014;18(34):43-56.

41. Smits S, Tamayo SP, Ibarra V, Rojas J, Benavidez A, Bey V. Gobernanza y sostenibilidad de los sistemas de agua potable y saneamiento rurales en Colombia. Bogotá: BID; 2012.

42. Ministerio de Ambiente, Vivienda y Desarrollo Territorial. Política pública de abastecimiento de agua y saneamiento básico para la zona rural de Colombia. Bogotá: Ministerio de Ambiente, Vivienda y Desarrollo Territorial, Dirección de Agua Potable, Saneamiento Básico y Ambiental; 2004.

43. Departamento Administrativo Nacional de Estadísticas. Gran Encuesta Integrada de Hogares. Bogotá: Dirección de Metodología y Producción Estadística; 2008.

44. Paredes N. El derecho a la salud en Colombia. En: Franco-Agudelo S, editor. La salud pública hoy: Enfoques y dilemas contemporáneos en salud pública. Bogotá: Universidad Nacional de Colombia; 2003. p. 287-342.
45. Ospina JM, Manrique FG, Ariza NE. Salud, ambiente y trabajo en poblaciones vulnerables: los cultivadores de papa en el centro de Boyacá. Revista Facultad Nacional de Salud Pública. 2008;26(2):142-152.

46. Ordóñez GA. Salud ambiental: conceptos y actividades. Revista Panamericana de Salud Pública. 2000;7(3):137-147.

47. Perea R. La educación para la salud, reto de nuestro tiempo. Educación XXI: revista de la Facultad de Educación. 2002;(4):15-40.

48. Nurgalieva ZZ, Malaty HM, Graham DY, Almuchambetova R, Machmudova A, Kapsultanova D, et al. Helicobacter pylori infection in Kazakhstan: effect of water source and household hygiene. American Journal of Tropical Medicine and Hygiene. 2002;67(2):201-206.

49. Bar AR, Oscherov EB, Milano AM. Hábitos y creencias sobre parásitos y enfermedades parasitarias en habitantes de Santa Ana de los Guácaras, Corrientes, Argentina. Boletín de Malariología y Salud Ambiental. 2009;49:127-133.

\section{FORMA DE CITAR}

Acosta CP, Benavides JA, Sierra CH. Análisis cualitativo del deterioro de la calidad del agua y la infección por Helicobacter pylori en una comunidad de alto riesgo de cáncer de estómago (Cauca, Colombia). Salud Colectiva. 2015;11(4):575-590.

Recibido: 23 de febrero de 2015 | Versión final: 17 de julio de 2015 | Aprobado: 20 de agosto de 2015

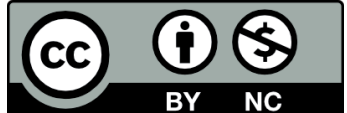

Este obra está bajo una licencia de Creative Commons Reconocimiento-NoComercial 4.0 Internacional. Reconocimiento - Permite copiar, distribuir y comunicar públicamente la obra. A cambio, se debe reconocer y citar al autor original. No Comercial - Esta obra no puede ser utilizada con finalidades comerciales, a menos que se obtenga el permiso. 